\title{
La incorporación de la regla variable en la enseñanza de E/LE
}

\author{
MARCIAL TERRÁDEZ GURREA \\ Universidad de Valencia \\ marcial.terradez@uv.es
}

\begin{abstract}
Resumen: Desde hace algunos años se viene insitiendo en la conveniencia de incorporar la información sociolingüística en la didáctica de una lengua. En el ámbito anglosajón ya se habla en ocasiones de la «gramática sociolingüística», y existen algunos intentos de enriquecer la información gramatical con datos variacionistas. Sin embargo, en el caso del español pocas veces se atiende a esa información, más allá de la necesidad de atender a las diferencias de registro a la hora de enseñar español a los alumnos extranjeros. Presentamos una propuesta de incorporación de la variable sociolingüística a la enseñanza de ELE, retomando la diferencia de Bernstein entre código restringido y código elaborado, y relacionando todo ello con nuestra propia investigación sobre el diferente uso del léxico según los estratos socioculturales. Nos centraremos en tres elementos léxicos: los adverbios en -mente, los sustantivos abstractos y los sustantivos clasificativos, mostrando cómo, a diferencia de lo que habitualmente se realiza en clase de ELE, los datos sobre el español hablado nos muestran que sería conveniente graduar didácticamente esos elementos, enseñando varios de ellos en los niveles superiores de ELE.
\end{abstract}

Palabras clave: Regla variable, Sociolingüística, variación léxica, frecuencias léxicas

\begin{abstract}
For some years the convenience of incorporating sociolinguistic information into the teaching of a language has been reiterated. In the Anglo-Saxon context, «sociolinguistic grammar» is discussed occasionally, there have been some attempts to enrich grammatical information with variational data. However, in the case of Spanish, this information is seldom addressed, beyond the need to address differences in register when teaching Spanish to foreign students. We present a proposal to incorporate the sociolinguistic variable into the teaching of SSL, taking Bernstein's difference between a restricted code and an elaborated code, and relating all this to our own research on the different use of the lexicon according to the socio-cultural strata. We will focus on three lexical elements: adverbs in -mente, abstract nouns and classifying nouns, showing, unlike what is usually done in a SSL class, how spoken Spanish data show us that it would be advisable to didactically classify these elements, teaching several of them in higher levels of SSL.
\end{abstract}

Key words: Variable rule, Sociolinguistics, lexical variation, lexical frequencies 


\section{Introducción}

Si bien la Sociolingüística no atiende en sus inicios a la vertiente didáctica, en los últimos años han aparecido una serie de trabajos que inciden en las aportaciones de esta disciplina a la didáctica de la lengua.

En el ámbito español, esos trabajos son deudores, de alguna manera, del artículo de Moreno Fernández (1994), quien plantea algunas de las aportaciones de la Sociolingüística para la enseñanza de lenguas. Posteriormente, otros autores como Carbonero Cano (2003), Caballero y Cuadrado (2007) o Lomas y Tusón (2012) han incidido en la relación entre Sociolingüística y didáctica.

No en vano, Moreno Fernández (1994: 111) señala que cuanto mejor se conozca el funcionamiento de una lengua y el uso de esa lengua en su contexto social, en mejores condiciones estaremos para afrontar su enseñanza, y eso es precisamente lo que nos aportan los estudios sociolingüísticos, un mejor conocimiento del funcionamiento de la lengua.

Un intento de utilizar los hallazgos de la Sociolingüística para una mejor enseñanza de la fonética lo podemos encontrar en Fasold (1984), quien afirma que si, como es habitual, los sonidos difíciles de la segunda lengua son más fáciles de pronunciar en unos contextos fonéticos que en otros, es conveniente estructurar los programas de tal forma que los sonidos nuevos que presentan una dificultad mayor sean introducidos y practicados, en primer lugar, dentro de los contextos que facilitan la articulación de ese sonido. Una vez que los estudiantes dominan la realización del sonido en esos contextos, se pasa a los contextos que lo favorecen en menor medida. Adamson (1988) piensa que estos principios se pueden aplicar a la sintaxis.

En definitiva, los análisis sociolingüísticos intentan cuantificar en qué medida influyen factores lingüísticos (contexto, función, etc.) ${ }^{1}$ y extralingüísticos (edad, nivel de instrucción, etc.) sobre los fenómenos variables, determinando cuál es la probabilidad de que esos fenómenos tengan una manifestación concreta en unas condiciones lingüísticas y sociales concretas. Lo que en Sociolingüística se denomina «regla variable» sería, por tanto, el estudio de la incidencia de una serie de factores sobre la posibilidad de aparición de un determinado elemento lingüístico (Moreno Fernández, 1994: 137). En este artículo defenderemos la conveniencia de la incorporación en la didáctica de la lengua de los datos que la Sociolingüística variacionista nos aporta, y ofreceremos alguna propuesta para la didáctica de léxico en la enseñanza del español como lengua extranjera.

\section{Nuestro estudio}

En este trabajo nos centramos en la variedad léxica del lenguaje, e intentaremos mostrar algunas vías para la utilización de la regla variable en la didáctica del léxico.

\footnotetext{
${ }^{1}$ Entendemos por «factores lingüísticos» aquellos elementos que tienen una incidencia sobre el lenguaje utilizado, y que no pertenecen a las características sociales o personales del hablante (Moreno Fernández, 1994)
} 
Estudiaremos la variable social «grado de instrucción» ${ }^{2}$, y utilizaremos datos procedentes de la investigación recogida en Terrádez Gurrea (2013). A partir del «grado de instrucción» estudiaremos el léxico empleado frecuentemente por los hablantes de los niveles socioculturales alto y bajo, con objeto de mostrar las diferencias entre ambos grupos sociales.

Nuestro planteamiento se acerca a la teoría del déficit de Bernstein (1964), quien sostiene que los hablantes de un nivel social alto disponen de un código elaborado, frente a los hablantes del nivel social bajo, que tienen a su disposición un código restringido.

El código elaborado se caracterizaría, entre otros aspectos, por un orden gramatical adecuado, un uso variado de conjunciones y cláusulas subordinadas, una elección cuidada de adjetivos y adverbios, un uso del lenguaje conceptual, etc., mientras que el código restringido presenta características como un uso frecuente de interjecciones, un lenguaje gramaticalmente sencillo, un uso restringido de adjetivos y adverbios, etc.

Para nuestra investigación, hemos analizado las palabras clave de los hablantes de cada uno de los dos niveles socioculturales objeto de estudio. Por palabras clave entendemos aquellos vocablos que aparecen con mayor frecuencia en el nivel social estudiado, en referencia a las palabras frecuentes del corpus general ${ }^{3}$.

Las palabras más utilizadas por los hablantes del nivel sociocultural alto se pueden dividir, entre otros, en los siguientes grupos:

1) Adverbios en -mente: realmente, absolutamente, fundamentalmente, evidentemente, básicamente

2) Sustantivos abstractos (acabados en -ad, -ez y -ción): prestación, atención, niñez, actividad, facilidad, inspección, verdad

3) Sustantivos clasificativos: nivel, especie, serie, aspectos

Por otra parte, las palabras que aparecen con frecuencia mayor de la esperada en los hablantes del nivel sociocultural bajo son:

1) Diminutivos: chiquillo, chiquilla

2) Algún valencianismo: diu, ara

3) Apelativos: hija, hombre, home, nena

4) Interjecciones: eh, uy, ah, ajá

5) Vulgarismos ${ }^{4}$ : to, pos, verdá

6) Deixis personal de primera persona: yo, digo, voy, hago, pongo

\footnotetext{
${ }^{2}$ La adscripción del nivel sociocultural al cual pertenece un hablante es un aspecto complejo y ampliamente debatido en los trabajos de Sociolingüística. En este artículo seguiremos lo planteado en la investigación que se recoge en Terrádez Gurrea (2013), y que consiste en asociar "nivel sociocultural" con «grado de instrucción». Para una discusión detallada sobre estos términos puede consultarse Moreno Fernández (1994: 52-65)

${ }^{3}$ Para una explicación más detallada del método para extraer las palabras clave de un corpus, consultar Terrádez Gurrea (2013).

${ }^{4}$ La noción de «vulgarismo» es controvertida. Aquí utilizaremos una idea de vulgarismo relacionada con aquellas formas que por su fonética se desvían de la norma del español estándar.
} 
En las listas anteriormente presentadas puede observarse que las palabras clave del nivel social alto están relacionadas con un estilo discursivo abstracto y, en cierta medida, argumentativo, mientras que el léxico frecuente de los hablantes del nivel bajo podemos asociarlo a una mayor inmediatez en el habla, así como a un estilo que podemos denominar como «emotivo».

A continuación, vamos a mostrar algunos ejemplos de nuestro corpus para observar los diferentes recursos léxicos de los hablantes de los dos niveles sociales estudiados en contextos discursivos similares:

\subsection{Nivel sociocultural alto ${ }^{\frac{5}{}}$}

(1) A: ¿hay muchas depresiones/ ahora?

B: sí/ fundamentalmentee/ la mayoría de gente que vienee es con problemas de depresion/ y por cierto/ tenemos bastante gente que pertenecen a la enseñanza/ ¿eh? al mundo de la enseñanza/ no tanto como alumnos sino como profesores
A: [¿ y cuál crees tú]
B: [hay- hay bastantes]

A: que son las causas?

B: (suspiro) (3") para mí fundamentalmente ess el tipoo de sociedad en la que estamos viviendo/ quiero decir hay muchoo- hay mucho aislamiento ee/ se ha perdido mucho/ ee sobre todo/ y volviendo otra vez a la genética yo creo que laa- los latinos/ o la cultura mediterránea/ ee tenemos una determinada forma dee- de ser/ y de VIVIR/ ee y de PENSAR también

(2) A: muy bien Maria/ hablemos ahora sobre tu tiempo libre// ¿tienes tiempo libre?

B: (chasquido) sí/ tengo tiempo libre (risas)

A: ¿y en qué lo dedicas- a qué lo dedicas? o ¿en qué lo gastas?

B: pues fundamentalmente en leer// que es unaa- una de mis aficiones/ eso más en- entre semana/ ee y luego los fines de semanaa ee a mí me gusta mucho eel- el cine/

\subsection{Nivel sociocultural bajo}

(3) A: [y tú ¿qué] aficiones tienes?/ ¿como gastas tu tiempo libre?

B: ¿mi tiempo libre?/ pues me gustaría muchísimo ir al cine/ porque me encanta el cine/// suelo leer bastante/// sobre todoo tipo dee- de novelas así policiacas me encantan

(4) A: ¿y en el pueblo hay muchos inmigrantes?

B: desde hace unos años sí/ sobre todo en invierno/ porque vienen para la temporada de la naranja/ yo he tenido roce con algunos al estar en el- en un bar/ y la verda(d) es que chicos trabajadores/ ee en situaciones muy pre- viviendo muy precarias/ trabajando por el mita(d) del jornal que un- de uno de aquí del pueblo

\footnotetext{
${ }^{5}$ En los ejemplos que vamos a mostrar a partir de ahora, señalamos con una A las intervenciones del entrevistador, y con una $\mathrm{B}$, las de los informantes.
} 
Vemos que los hablantes del nivel bajo no utilizan el adverbio acabado en -mente como elemento de focalización, sino que prefieren estructuras como «sobre todo». De hecho, no hemos encontrado ningún ejemplo de «fundamentalmente» en los discursos de los hablantes del nivel sociocultural bajo.

\subsection{Nivel sociocultural bajo}

(5) A: ¿qué harás cuando te jubiles?

B: pues es una de miss grandes preocupaciones// yo tengoo muy claro que cuando una persona se jubila y no se busca una actividad alternativa// ee CAE en una depresión/ que le puede llevar/ pues aa la- al abandono/ e incluso en muchos casos a la muertee prematura

(6) B: la vivienda rural ha cambiado muchísimo/ en los últimos años/ de tal manera que aquellas casass/ quee existían enn mi infancia/ dondee a vecess ee la parte principal de la casa era el- el establo/ como llamaban allí la cuadra/ para los animales/ porque esos animales eran/ un poco el motor dee- de toda la actividad económica/ con lo cual les hacían todas las tareas agrícolas etcétera

\subsection{Nivel sociocultural bajo}

(7) A: ya pero tú ahora ¿como te lo montarías/ llegado ese caso de la jubilación?

B: puees/ a pasear por aquí/ pasear por allá/ pescar yy- o a irme de caza o aa- no sé// hacer cosas en casa// estar con los amigos// irme por ahí a pasear o a pescar/ a ver- a ver la playa/ a ver el paseo/ las obras// lo que fuera

(8) A: claro que si Emilio/ bueno la jub- la jubilación está ya cerca

B: sí// [está cerca]

A: [¿qué-] qué hay previsto?

B: ¿previsto?/ previsto viajar/ casi casi la de todos (risas)/ viajar/ sí sí sí sí

A: [más cosas]

B: [no no]/ quedarme a la bartola ee a la tumbona/ no no/ yo prefiero estar activo de una forma o de otra/ pero no trabajando ¿eh?/ no trabajando como tal/ sino tener jobis// me encanta el baile

En los ejemplos anteriores se puede observar que, mientras los hablantes del nivel sociocultural alto utilizan palabras generalizadoras como «actividad», lo informantes del nivel bajo prefieren utilizar ejemplos concretos, sin hacer referencia a ningún elemento léxico que englobe esos ejemplos. Como hemos señalado al comentar las listas de palabras frecuentes de cada nivel social, los hablantes del nivel social bajo muestran un menor grado de generalización o de abstracción en sus conversaciones.

\subsection{Nivel sociocultural alto}

(9) B: la habitación dormitorio y al baño/// en el mismo salón/ en un lateral/ de- mm de la piedra hay unaa escalera que sube a una especie de estudio/ a un altillo que es un estudio// construido también sobre el muro/ 
(10) B: bueno eenn- fiestas ee/ sí/ recuerdo fiestas/ bueno fiestas populares/ de ambiente/ ee recuerdoo/ y el otro día loo recordé además porque vi una fotografia// una especie de procesión cabalgata que se hacia en Nules// que se llamaba la festa de les barraquetes

\subsection{Nivel sociocultural bajo}

(11) B: primera planta ee está la cocina// un cuarto de aseo/ yy- y el comedor// tenemos un sótano/ en el sótano ee es donde aparcamos el coche/ porque es como si fuer- es como una calle/ BAJO/ que entramos todos los vecinos// pero ee cada- cada casa tiene su cochera individual/ en vez de tener la calle por arriba/ pues se tiene por abajo

(12) B: el corral/// yy/ bueno/ allí todos los vecinos pues se conocían ¿no?/ porque eran// tipoo/ habitaciones ¿no?// donde vivían pues/ cada familia ¿no?/ y allí nos conocíamos todos// había un lavadero común///(2") ee luego pues eso/ saliamos todos los niños allí/ ee- en el medio del corral era// como uun- como unn

C: de tierra

B: de tierra/ si/ de tierra

$\mathrm{C}$ : como [un parque]

B: $\quad$ [como un] parque de tierra/ y alli pues jugabamos todos/ llegaban mis primos/ jay!/ me encantaban que vinieran mis primos

En estos ejemplos comprobamos, una vez más, la diferencia en el estilo discursivo de los hablantes de ambos niveles sociales, pues los informantes del nivel socicultural alto utilizan una palabra como «especie», un sustantivo clasificativo que, como tal, expresa un grado de abstracción notable. Sin embargo, los hablantes del nivel social bajo prefieren emplear paráfrasis del tipo «como si fuera», «como un....». De nuevo aparece la diferencia entre una conversación con el uso de abstracciones y generalizaciones y otra conversación más ligada a lo concreto e inmediato.

\subsection{Nivel sociocultural alto}

(13) B: los co- los clásicos que yo he conocido/ que han investigado determinadas cuestiones/ dee pequeños detalles/ ee pequeñas monografías/ de determinados aspectos/ de- no di- de la cultura clásica/ de la filología clásica// que NO INTERESAN a nadie

(14) B: ¿qué aspectos son importantes?/ pues bueno/ pues los aspectos mas importantes de mi vida/ ha sido/ eel ejemplo de los padres// el gran sacrificio/ ee que en aquellos entonces/ que eran muy diferentes de los de ahora

\subsection{Nivel sociocultural bajo}

(15) B: ¿como lo convencería? pues// asentándolo y hablándole de las cosas malas que puede haber// yy- y llevándolo a un sitio// donde se puede ver 
(16) B: conforme has ido quedando para las fechas de entrega/ que es una de las cosas más importantes que hay/ en un taller pequeño/ o seaa que cumplas con el cliente/ si le dices el jueves/ que el jueves tenga el coche

Observamos en los anteriores ejemplos que, frente a la tendencia de los hablantes del grado de instrucción alto a utilizar palabras generalizadoras, como «aspectos», los hablantes del nivel social bajo prefieren actualizar proformas léxicas, como «cosa».

\section{Reflexiones y conclusiones}

¿Qué conclusiones podemos extraer de los ejemplos anteriormente señalados? Como hemos podido comprobar, los resultados de nuestro estudio confirman los planteamientos de Bernstein. Los hablantes del nivel sociocultural alto reflejan en sus conversaciones la presencia de un código lingüístico elaborado, utilizando con frecuencia elementos léxicos abstractos, generalizadores y argumentativos, mientras que los informantes que pertenecen a un nivel social bajo muestran un código restringido, lleno de palabras comodines, circunloquios y léxico emotivo.

De los anteriores ejemplos puede inferirse la necesidad de incorporar la información sociolingüística en la gramática didáctica, principalmente en la gramática dirigida a los alumnos de ELE, fundamentalmente en el sentido de reservar aquellos elementos léxicos propios del código elaborado para niveles altos de enseñanza de español como lengua extranjera $(\mathrm{C} 1$ o $\mathrm{C} 2)$.

$\mathrm{Si}$ revisamos el Plan Curricular del Instituto Cervantes (http://cvc.cervantes.es/ensenanza/biblioteca_ele/plan_curricular/indice.htm), podemos comprobar que no hay una decisión de graduar los elementos lingüísticos que se enseñan (por ejemplo, aquellos elementos que se han reseñado en este artículo, como los adverbios acabados en -mente o los sustantivos clasificativos). Nuestra propuesta sería graduarlos en función del código (restringido o elaborado) al que pertenecen, y utilizar esa graduación a la hora de decidir qué aspectos de la lengua van a enseñarse a los estudiantes en función de su nivel (A1, B2...).

Así, por ejemplo, consideramos que no resulta adecuado explicar todos los adverbios acabados en -mente en un mismo bloque temático, sino que deberíamos reservar la presentación de las funciones discursivas y semánticas de los adverbios en -mente apenas utilizados en el habla por los hablantes de niveles socioculturales medio y bajo para aquellos alumnos de ELE que presentan un nivel avanzado en su conocimientos de la lengua. Y lo mismo podría aplicarse en el caso de algunos sustantivos clasificativos.

En definitiva, se trata de enseñar el vocabulario real de los hablantes de una lengua a aquellos estudiantes que están aprendiendo español.

\section{Bibliografía}

AdAmson, H. (1988). Variation Theory and Second Language Acquisition. Washington, D.C.: Georgetown University Press.

Bernstein (1964): «Elaborated and Restricted Codes: Their Social Origins and Some Consequences», American Anthropologist, 66, 55-69. 
Caballero Murillo, M. y Cuadrado Gordillo, I. (2007). «La sociolingüística y la enseñanza de la lengua y la cultura de origen», Revista de Educación, 342, 503-527.

CARbonero CANO, P. (2004). «Repercusiones de la Sociolingüística Andaluza en la didáctica de la lengua», Cauce, Revista de Filología y su Didáctica, 27, 35-48.

FASOLD, R. (1984). «Variation theory and language learning». En P. Trudgill (ed.), Applied Sociolinguistics (pp. 245-261), London: Academic Press.

Lomas Osoro, C. y Tusón Valls, A. (2012). «Lengua, cultura y sociedad. Una mirada sociolingüística a la enseñanza de la lengua». Textos de Didáctica de la Lengua y de la Literatura, 60, 77-91.

Moreno Fernández, F. (1994). Principios de sociolingüística y sociología del lenguaje. Madrid: Ariel.

Moreno FERnÁndeZ, F. (1994): "Aportes de la sociolingüística a la enseñanza de lenguas», Revista de Estudios de Adquisición de la Lengua Española, 1, 107-135.

TERrÁDez GurReA, M. (2013): «Estratificación y frecuencias léxicas», en Gómez Molina, J.R. (coord.). El español de Valencia. Estudio sociolingüístico (pp. 247-276). Peter Lang: Suiza. 